\title{
Strategies to Improve Tuberculosis Counseling Services
}

\author{
*Ms.Nandhini \& Prof. KaralineKarunagari
}

\section{Abstract:}

Tuberculosis is an acute or chronic infection caused by Mycobacterium tuberculosis.Tuberculosis (TB) is one of the top 10 causes of death worldwide. In 2015, 10.4 million people fell ill with TB and 1.8 million died from the disease (including 0.4 million among people with HIV). Over 95\% of TB deaths occur in low- and middle-income countries. Counseling plays a significant role in providing TB patients with complete and accurate knowledge about the nature of $T B$, its symptoms, mode of transmission and how to manage the disease (prevention of transmission, managing drugs' side effects etc.).In addition they require lot of psychological support and effective treatment,follow up services are needed to promote their health and reduce the incidence of tuberculosis in the community.

Keywords: Tuberculosis, Strategies and Counseling services

\section{INTRODUCTION}

Tuberculosis is an acute or chronic infection caused by Mycobacterium tuberculosis. TB is characterized by pulmonary infiltrates, formation of granulomas with caseation, fibrosis, and cavitation. People living in crowded and poorly ventilated conditions and who are immunocompromised are most likely to become infected. Tuberculosis (TB) is one of the top 10 causes of death worldwide. In 2015, 10.4 million people fell ill with TB and 1.8 million died from the disease (including million among people with HIV).
Over $95 \%$ of TB deaths occur in low- and middle-income countries.In 2015, an estimated 1 million children became ill with $\mathrm{TB}$ and $170 \quad 000$ children died of TB (excluding children with HIV).TB is a leading killer of HIV-positive people: in 2015, 35\% of HIV deaths were due to TB.

Globally in 2015, an estimated 480000 people developed multidrug-resistant TB (MDR-TB).TB incidence has fallen by an average of $1.5 \%$ per year since 2000 . This needs to accelerate to a $4-5 \%$ annual decline to reach the 2020 milestones of the "End TB 
Strategy". An estimated 49 million lives were saved through TB diagnosis and treatment between 2000 and 2015.Ending the TB epidemic by 2030 is among the health targets of the newly adopted Sustainable Development Goals.

\section{WHO pursues 6 core functions in addressing TB.}

- Provide global leadership on matters critical to TB.

- Develop evidence-based policies, strategies and standards for TB prevention, care and control, and monitor their implementation.

- Provide technical support to Member States, catalyze change, and build sustainable capacity

- Monitor the global TB situation, and measure progress in TB care, control, and financing.

- Shape the TB research agenda and stimulate the production, translation and dissemination of valuable knowledge.

- Facilitate and engage in partnerships for TB action.

\section{Overcoming TB Treatment Barriers through Counseling}

Counseling plays a significant role in providing TB patients with complete and accurate knowledge about the nature of TB, its symptoms, mode of transmission and how to manage the disease (prevention of transmission, managing drugs' side effects etc.).In addition they require lot of psychological support as there is lot of stigma associated with TB and people with TB disease do not access the treatment for the fear of being discriminated and isolated within their community.TB patients and their families also need to be educated regarding consequences of incomplete /inadequate TB treatment (e.g. development of Drug Resistant TB) and require ongoing counselling to motivate them to strictly adherence to TB drug regimen.Helping clients overcoming barriers to completion of TB treatment is the foundation of effective counseling.

\section{PHASES/STRATEGIES OF TB} COUNSELING SERVICES:

$>$ Phase 1-Pre Diagnosis Counseling

Case Detection

$>$ Phase 2- Initial Counseling

$\leftrightarrow$ TB Diagnosis \& Initiation of Treatment

$>$ Phase 3- On going Counseling

Continuation \& Completion of Treatment

ROLE OF NURSE IN THE PHASES OF TB COUNSELING

Phase 1-Pre Diagnostic Counseling (Case Detection)

Pre diagnosis phase of counseling with a client is quite crucial as it would set the tone for the patient-provider relationship that will continue to develop throughout diagnosis and treatment. Great emphasis should be placed on forming rapport by showing respect and warm friendly behavior.

The first meeting with a client usually is when he or she is referred by the primary care provider to the TB clinic. The provider should clearly define the objectives of his or her conversation with the client and plan the meeting so there is enough time to discuss the important aspects. It is also important to give the client, time to think. If he or she cannot answer the provider's questions or gives unclear or incorrect responses, we should help the client by clarifying the information again and asking additional questions. Also the client should be encouraged to ask his/her own questions \&concerns.Similarly, at the end of the session, we should summarize the main points discussed and ensure that the client has understood all the information. 
The objectives of counseling at this stage are:

To develop rapport with the client

To collect complete medical \& social history

To discuss the potential TB diagnosis

* To provide client with basic information on TB,various diagnostic tests etc.

$\approx$ To reassure client that TB is curable and is Free of cost under the Govt. health system

To motivate client to take the TB tests

\section{Phase 2-Initial Counseling (TB Diagnosis \& Treatment Initiation)}

This phase starts when the client comes for the test results and TB treatment is initiated for those who test positive. Initial phase counseling should focus on

$\rightarrow$ Explanation of the TB diagnosis

$\rightarrow$ Understanding patient's perceptions about TB and concerns about being diagnosed with TB

$\rightarrow$ Explanation of the following:

1. TB and its symptoms, emphasizing that TB is a curable disease

2. TB treatment regimen: duration, intensive phase of treatment, supportive phase of treatment

3. Drugs and doses used to treat TB

4. Possible side effects of medication and what to do

5. The importance of taking medications regularly for the full course of treatment and options available for DOT/treatment support

6. Frequency and importance of sputum examination and understanding of sputum results

7. Awareness of the symptoms and infectiousness of tuberculosis \& prevention of spreading TB to other.
8. Importance of direct observation of every dose in the intensive phase.

9. The importance of screening family members for $\mathrm{TB}$

10. Assessment of available supports and potential barriers to treatment adherence and completion

11. Patient concerns about the treatment and follow-up care

12. Next steps, referrals and schedule for next visit

It is important to reinforce the fact that TB is curable and is free of charge under Govt Health system.It is very important to pay careful attention to the patient's emotions and help him or her deal with the shock of learning that he or she has TB. Having TB carries a stigma for many people and may lead to feelings of shame, fear of social rejection, or fear of the loss of a job. The main task of us as at this stage is to give the client a chance to talk, listen actively to his or her concerns, and address questions and concerns regarding TB treatment.

Understanding the patient's perspective and understanding regarding $\mathrm{TB} \&$ its treatment, is an important step toward ensuring adherence. Because misinformation and misconceptions about TB increase the likelihood of nonadherence, it is important to identify and address these issues in the beginning of the treatment.

Not all of these questions should be asked at once, but we should try to ask several key questions based on the patient's reactions to the diagnosis that can identify the most important issues for the patient. It is important during the initial counseling of a TB patient to explain the crucial need of knowing his or her HIV status and encourage the patient to get tested for HIV. 
We should use audio visual materials to explain key points, should show the actual pills the client will be taking, to reinforce spoken information. The information also should be supplemented with simple, easy- tounderstand written materials that the patient can take away from the visit.

\section{HIV-TB Counseling}

Counseling \& motivating TB clients for HIV testing is one of the important aspects of initial TB counseling. It is important to emphasize during the counseling that although most patients with $\mathrm{TB}$ do not have HIV, there are still a large number of TB patients who may be suffering from undiagnosed HIV infection. Since, anHIVpositive person is at much higher risk of developing active $\mathrm{TB}$, timely diagnosis would result in initiating prompt initiation of treatment.

The nurse should address patient's concern regarding HIV associated stigma and assure him/her of the confidentiality and that the testing will not take place without client's consent; also that the post-test counseling would be undertaken irrespective of the HIV status.

The client must be strongly encouraged to return to the nurse with the HIV results as in case of positive HIV results, he/she would again need counseling \& psychological support to cope with implications of having dual infection. The client would require ongoing counselling on treatment adherence and Positive living as well.

\section{Phase 3-On going counseling (Continuation \& Completion of Treatment)}

It is important for the medical provider to offer ongoing counseling during regularly scheduled visits by reinforcing the messages already provided and encouraging the patient to continue treatment to cure.
The main objectives for counseling during the outpatient phase of treatment include:

To check whether the patient is taking medications correctly and help the patient address any barriers to adherence

To emphasize the importance of treatment continuation and completion even when the patient feels much better

To provide emotional support to the patient and refer him or her for other support as needed

毁 To check for possible side effects

To strengthen the patient's motivation to continue and complete the treatment

Adherent patients tend to understand the causes of TB and its treatment; however, patients with active disease who are initially adherent may begin to feel well after a few weeks of treatment and may then forget their medication or believe that it is no longer needed.

Therefore, even patients who have followed the regimen early in the treatment should be monitored and given assistance to ensure continued adherence after symptoms begin to subside.

It is important to remember that the nurse should limit the amount of information to present in any one visit. Topics should be organized in the order of importance. Communication with the patient should always be two way. Nurse should elicit feedback and questions from the patient to ensure that the message sent was received and understood.

Ten Points Counseling Tool on TB.

Please ensure that the following has been covered during the counseling session

1 . TB is an airborne infection, caused by a germ - Mycobacterium tuberculosis. It is estimated that $40 \%$ of the Indian population is infected with TB. 
2. TB disease is the most common Opportunistic Infection in HIV disease, the most common cause of morbidity and leading cause of death in PLHIV.

HIV increases the risk of progression from TB infection to TB disease

PLHIV infected have a 50\% lifetime risk of developing TB disease, where as a HIV negative person infected with $\mathrm{TB}$ has a $10 \%$ life-time risk of developing TB disease.

3. A person contracts TB infection from an open case of TB (usually a sputum smear positive Pulmonary TB case). However infection with $\mathrm{TB}$ does not necessarily mean that the infected person would develop TB disease. An infected person develops disease when his/her immunity declines.

4. Prolonged cough, for 2 weeks or more, can be TB disease and therefore it is essential to consult a doctor and get sputum examined to rule out TB.

5. Two sputum smear examinations are necessary for the diagnosis of pulmonary TB. During the course of treatment the progress is monitored by means of follow up sputum examinations every 2 or 3 months until the end of treatment.

6. Anti TB drugs are provided in PatientWise drug Boxes, which ensures that the full course of treatment is available at the start of treatment.

7. Full course of treatment is necessary to be cured of TB, though a patient starts feeling better after taking anti- $\mathrm{B}$ treatment for a short duration. Cure from TB can only be ensured by taking complete and regular treatment, whether a patient is infected with HIV or not

8. Maintaining Cough Hygiene and other Infection Control practices at home is important to prevent TB transmission to others at home

9. Sputum microscopy and treatment services for TB are available free of cost through the Revised National TB Control Program (RNTCP)
Treatment is provided at a place near the patient's home which is convenient and acceptable to the patient and accountable to the system.

As per RNTCP policy, before treatment initiation, there is a process of address verification, by which the program ensures that any patient who is late for their treatment can be retrieved and brought back onto treatment. Hence, patients must disclose their correct address to the staff providing TB treatment.

RNTCP takes upon itself the responsibility of curing the TB patient. RNTCP treats all patients irrespective of HIV status.

10 The ICTC maintains patient's confidentiality and does not disclose the HIV status of the patient to the personnel providing RNTCP services.

The patient should voluntarily disclose his/her HIV status to the treating physician for correct/ better categorization and also to get benefit of prophylactic/ treatment options available for him

\section{Addressing stigma and discrimination}

It is very difficult to measure stigma, it can be observed in the form of discrimination. The TB patient may either be isolated, kept secluded or there could be self-withdrawal by the patient. Discrimination towards TB patients can be observed in any of the following forms:

* Female patients are sent to parental house for treatment

* Men avoid letting anyone know about the disease at work place for the fear of being isolated

* Parents of young girls do not want any one from the health centre to visit their home

The following activities may be done for reducing discrimination towards TB patients

* Counseling and motivation support TB patient during the course of treatment

* Informing communities about curability of disease 
* Involving cured persons for sharing their experience and how they have been fully cured with DOTS

* Motivation and reassurance while interacting with TB patients

* Providing patients and families with information material

* Getting eminent people to speak about TB Role of Nurses

* To participate in the IEC activities at the community level to raise awareness regarding TB disease

* To counsel \& refer clients having signs \& symptoms of TB infection for early diagnosis and treatment initiation

* To counsel and support TB patients throughout the treatment duration and motivate them to complete the treatment.

\section{CONCLUSION:}

Counseling plays a significant role in providing TB patients with complete and accurate knowledge about the nature of TB, its symptoms, mode of transmission and how to manage the disease (prevention of transmission, managing drugs' side effects etc.).In addition they require lot of psychological support and effective treatment,follow up services needed to promote their health and reduce the incidence of tuberculosis in the community.

\section{REFERENCES:}

1. WHO Global Tuberculosis report(2017)

2. Indian Nursing Council, Training of Nurses on Tuberculosis care \& control in India Nurses' Mannual(2014).

3. WHO. Documentation for World Health Assembly

67.

http://apps.who.int/gb/ebwha/pdf_fil es/WHA67/A67_11-en.pdf (accessed Sep 18, 2016).

4. WHO. Global tuberculosis control: WHO report 2016 (WHO/HTM/TB/accessed sep 18, 2016.). Geneva: World Health Organization, 2016.

5. Global TB Programme, World Health Organization, Geneva, Switzerland. http://dx.doi.org/10.1016/S01406736(15)60570-0 (accessed Sep 18, 2016)

\section{INTERNATIONAL PEER REVIEW MEMBERS}

\author{
Mr. Allan Seraj \\ Senior Practice Educator in ICU \\ RoyalBrompton\&Harefield Hospital \\ NHS Trust, UK
}

\author{
Dr. Rachelle (Shelly) J. Lancaster \\ Associate Professor of Nursing \\ Oshkosh College of Nursing \\ University of Wisconsin \\ Oshkosh Wisconsin, USA.
}

